\section{and Functional}

Neurosurgery
Stereotact Funct Neurosurg 2021;99:329-342

DOI: $10.1159 / 000512111$
Received: July 21, 2020

Accepted: September 28, 2020

Published online: December 10, 2020

\title{
Technical Principles and Clinical Workflow of Transcranial MR-Guided Focused Ultrasound
}

\author{
Ying Meng a, b Ryan M. Jones ${ }^{a}$ Benjamin Davidson ${ }^{a}$ b Yuexi Huang ${ }^{a}$ \\ Christopher B. Pople ${ }^{a}$ Shanan Surendrakumar ${ }^{a}$ Clement Hamani ${ }^{a, b}$ \\ Kullervo Hynynen ${ }^{a, c, d}$ Nir Lipsman ${ }^{a, b}$
}

a Sunnybrook Research Institute, University of Toronto, Toronto, ON, Canada; bivision of Neurosurgery, Department of Surgery, University of Toronto, Toronto, ON, Canada; 'Department of Medical Biophysics, University of Toronto,

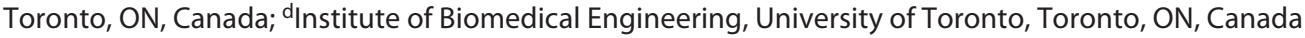

\section{Keywords}

MR-guided focused ultrasound $\cdot$ Blood-brain barrier

disruption · Thermoablation

\section{Abstract}

Transcranial MR-guided focused ultrasound (MRgFUS) is a rapidly developing technology in neuroscience for manipulating brain structure and function without open surgery. The effectiveness of transcranial MRgFUS for thermoablation is well established, and the technique is actively employed worldwide for movement disorders including essential tremor. A growing number of centers are also investigating the potential of microbubble-mediated focused ultrasound-induced opening of the blood-brain barrier (BBB) for targeted drug delivery to the brain. Here, we provide a technical overview of the principles, clinical workflow, and operator considerations of transcranial MRgFUS procedures for both thermoablation and BBB opening.

(C) 2020 The Author(s)

Published by S. Karger AG, Basel

karger@karger.com www.karger.com/sfn

Karger"

GOPEN ACCESS (c) 2020 The Author(s)

Published by S. Karger AG, Basel

This is an Open Access article licensed under the Creative Commons Attribution-NonCommercial-4.0 International License (CC BY-NC) (http://www.karger.com/Services/OpenAccessLicense), applicable to the online version of the article only. Usage and distribution for commercial purposes requires written permission.

\section{Introduction}

As of early 2020, over 50 institutions worldwide offer transcranial magnetic resonance-guided focused ultrasound (MRgFUS) as a less-invasive treatment option for thermoablation in the brain and $>10$ are involved in clinical testing of FUS combined with microbubbles for targeted drug delivery to the central nervous system (CNS) [1]. Other applications of FUS in the brain that are currently under clinical investigation include neuromodulation and ultrasound-assisted clot lysis (i.e., sonothrombolysis) $[2,3]$. Owing to the appeal and potential benefits of less-invasive surgical techniques, MRgFUS thermoablation has superseded previously developed lesioning approaches at many institutions, particularly for unilateral thalamotomy in the treatment of medication-refractory essential tremor (ET) [4-6]. With demonstration of the cost-effectiveness of transcranial MRgFUS relative to standard surgical treatments for ET, this trend is poised to continue for other intracranial ablative indications [7, 8]. Additionally, low-intensity pulsed FUS and circulating microbubbles can increase blood-brain barrier (BBB) permeability to enable localized drug delivery to the CNS and is a burgeoning field of clinical research built on a 
promising line of preclinical investigations since the initial feasibility was demonstrated in 2001 [9-15]. If shown to be safe and effective in a clinical setting, this technology may open opportunities to treat diverse neurological conditions for which the $\mathrm{BBB}$ is a major impediment to therapeutic delivery. Other techniques to bypass the BBB exist for enhanced CNS drug delivery. However, some of these techniques have shortcomings and risks that limit more widespread adoption, such as the heterogeneous $\mathrm{BBB}$ opening (BBBO) and off-target toxicity associated with intra-arterial mannitol $[16,17]$.

Over the past few years, there has been increasing interest in the use of FUS as a tool for modulating BBB permeability in a number of clinical indications, ranging from cancer to neurodegenerative disease. This article provides an overview of the basic principles of transcranial MRgFUS, the current state of the technology, treatment protocols, and existing limitations. We emphasize the methodology and nuances of nonthermal microbubble-mediated FUS procedures (e.g., BBBO) and highlight areas of improvement for the treatment team.

\section{State of Technology}

The currently available clinical FUS brain devices can be stratified into MR-guided (ExAblate Neuro; InSightec, Israel), neuronavigation-guided (NaviFUS, Taiwan), and implanted (SonoCloud, CarThera, France) systems. Two ExAblate Neuro systems are available, and these systems share the same peripheral electronics but operate at different frequencies: a high-frequency $(650-720 \mathrm{kHz})$ device for thermal ablation and a low-frequency (220-230 $\mathrm{kHz}$ ) device for nonthermal microbubble-mediated treatments. The high-frequency ExAblate Neuro system is currently the only FUS device approved for thermoablation in the brain. The low-frequency ExAblate Neuro system, along with NaviFUS and SonoCloud, is the clinicalprototype device for FUS and microbubble-mediated BBBO. The ExAblate Neuro and NaviFUS systems use extracorporeal FUS transducers, whereas the SonoCloud device is an unfocused ultrasound transducer that is implanted in the skull cavity [18]. Several laboratory FUS brain systems exist and are at various stages of development and testing [19-21].

This article focuses mainly on the ExAblate Neuro devices, which have been employed for both thermal and nonthermal FUS brain treatments, since most of the clinical experience to date has been based on these devices. The ExAblate Neuro system consists of a $30-\mathrm{cm}$ diameter hemispherical surface ("helmet") that is lined with 1,024 independent transducer elements for ultrasound delivery. The helmet is fixed to a specialized MRI bed and is attached to the patient's head using a stereotactic frame. The intervening space is filled with chilled degassed/deionized water, held in place by a rubber membrane fitted around the circumference of the head, which provides acoustic coupling and enables cooling of the scalp and skull during thermoablation procedures. Based on the prescribed target location, helmet orientation, skull properties (estimated from patient-specific CT images), intraoperative MRI data, and additional user input (e.g., nopass zones for intracranial calcifications and water membrane folds), the MRgFUS system calculates the necessary amplitude and phase for each individual transducer element to focus ultrasound through the skull bone to the prescribed target(s) [22]. In general, only a subset of the array may be active for a given sonication as elements associated with no-pass zones or high incident angles are disabled [23]. For thermal treatments, the transducer amplitude distribution is often set to achieve a uniform acoustic intensity density on the outer skull surface [24] to minimize skull heating effects [25]. For nonthermal treatments, during which skull heating is less of a concern, the transducer amplitude distribution is often set to ensure each array element contributes equally to the acoustic intensity at the focal spot [24]. As discussed in further detail below, intraoperative MRI is carried out to provide real-time treatment guidance and dedicated single-element acoustic detector monitor for any cavitation activity generated during the FUS exposures.

\section{Basic Principles: Thermal versus Nonthermal}

A critical responsibility of the treatment team is to promote efficient propagation or delivery of ultrasound into the brain such that the intended biological effect(s) occur at the target(s). Propagation of ultrasound into a medium is dictated by the acoustic impedance, which is related to the material density and sound speed. In passing through the skull, ultrasound is reflected, attenuated, and transmitted. At the interface separating 2 distinct media, a higher mismatch in acoustic impedance (e.g., from scalp to bone) results in increased reflected energy and consequently decreased ultrasound transmission (Fig. 1). Temperature elevation is the primary result of attenuation in the skull bone. Attenuation varies as ultrasound penetrates through different tissue, indicated by an attenuation coefficient of $13.0 \mathrm{~dB} / \mathrm{cm}$ for the skull and $0.6 \mathrm{~dB} / \mathrm{cm}$ 


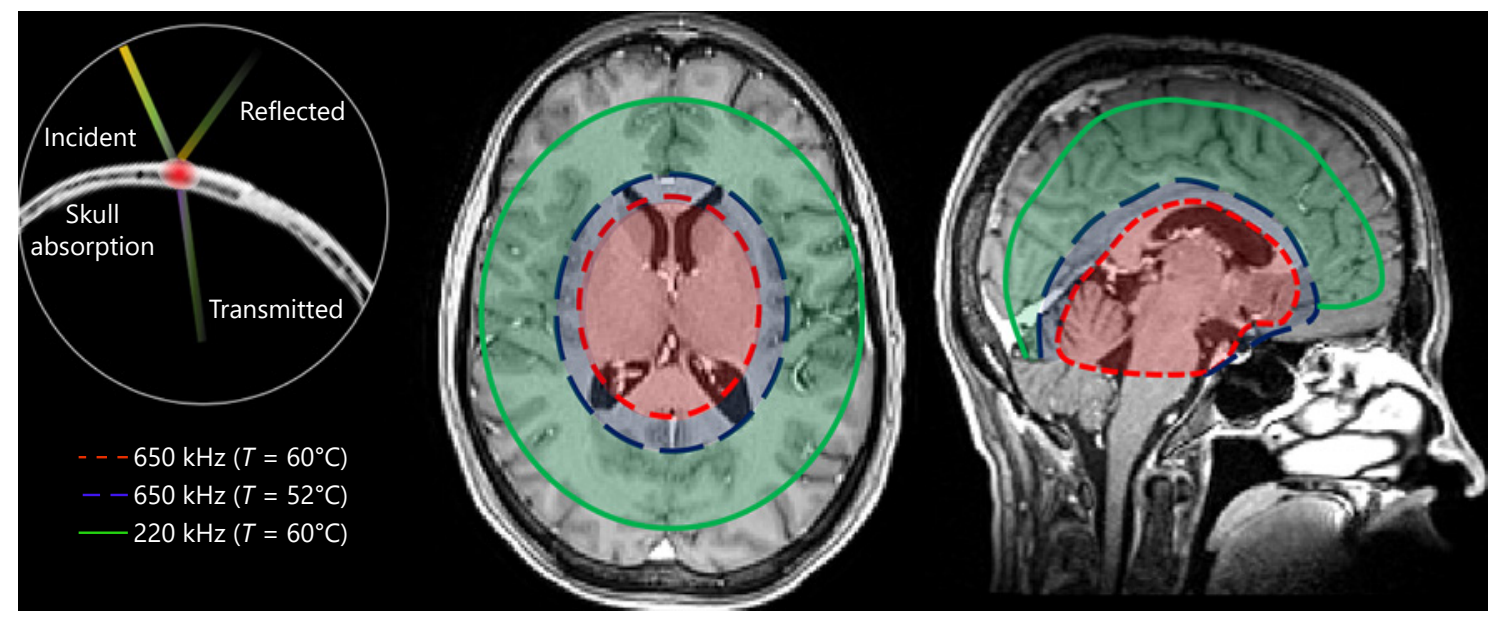

Fig. 1. Left: ultrasound is reflected, attenuated (absorbed and converted into heat), and transmitted as it passes through the skull. Reflections and attenuation within the bone contribute to reduced levels of transmission at the intracranial target, and variations in skull morphology (e.g., thickness) and material properties act to defocus the ultrasound beam. Right: treatment envelopes for a fixed peak focal temperature obtainable via existing hemispherical MRgFUS brain systems with different operating frequencies, adapted from 2013 Focused Ultrasound Foundation Brain Mini Workshop on Treatment Envelope Expansion [88]. MRgFUS, transcranial MR-guided focused ultrasound. for the brain at $1 \mathrm{MHz}$ [26]. For comparison, a 3-dB decrease translates to a $50 \%$ reduction in ultrasound intensity. In addition to attenuation, variations in skull thickness introduce aberrations to the ultrasound path, defocusing the ultrasound beam. Overall, ultrasound suffers from increased attenuation and distortion with rise in frequency [26].

Using a curved single-element transducer or a multielement phased array, focused ultrasound creates a tight high-pressure point with a sharp drop off outside the focus. Phased arrays permit electronic control over the beam geometry and direction, as well as provide the ability to mitigate skull-induced beam distortions, and thus provide increased flexibility relative to single-element transducers. The size of the focal region can be adjusted depending on the transducer design (e.g., geometry) and ultrasound parameters (e.g., operating frequency). The target brain region should ideally be spatially matched to the geometric focus of the transducer. Arrays can be mechanically positioned such that the focus is as close as possible to the target, after which the focus can be steered electronically through phasing within a smaller region. The low-frequency ExAblate Neuro system allows sonications within a sphere approximately $4 \mathrm{~cm}$ in diameter without repositioning the helmet. Overall, the treatment envelope is larger for the low-frequency versus high-frequency system, provided the helmet is repositioned to place the target within the treatment sphere. For high-

Transcranial MR-Guided Focused Ultrasound frequency applications, treatment outside of these zones is suboptimal because of decreased efficiency of ultrasound delivery, increased skull heating, and potential offtarget effects.

Thermal dose is an important concept in understanding FUS thermoablation applications in the brain. The biological effects resulting from thermal exposures depend on temperature as well as the exposure duration. For this reason, thermal dose was developed as a metric to evaluate the extent of tissue damage resulting from thermal exposures [27]. For a given exposure, thermal dose can be interpreted as the number of cumulative minutes at $43^{\circ} \mathrm{C}$ that would be required to achieve an equivalent biological effect. With $43^{\circ} \mathrm{C}$ chosen empirically from in vitro experiments, for every additional degree of temperature elevation, the time required for coagulative necrosis decreases by $50 \%$ [28]. In general, each target tissue has a different sensitivity to thermal dose though $240 \mathrm{cu}$ mulative equivalent minutes at $43^{\circ} \mathrm{C}\left(\mathrm{CEM}_{43}\right)$ appears sufficient to induce necrosis in all tissue types [29]. Data from MRgFUS thalamotomy procedures suggest the dose threshold is lower $\left(\sim 100 \mathrm{CEM}_{43}\right)$ in the human thalamus $[30,31]$. The bioheat transfer equation is often used to model heat transfer and was formulated by Dr. Harry Pennes [32], a psychiatrist in New York City, through experiments in the human forearm. Dr. Pennes introduced the importance of convective heat loss through perfusion as well as diffusion to surrounding structures, which ex- 
Table 1. Types of therapeutic focused ultrasound classified by ultrasound frequency, intensity, and use of microbubbles

\begin{tabular}{lll}
\hline Frequency & Intensity & \\
\cline { 2 - 3 } & low & high \\
\hline Low & $\diamond$ BBBO with microbubbles [44] & $\bullet$ Ablation [85] \\
High & $\bullet$ Neuromodulation [42, 58] & Thermoablation, for example, MRgFUS \\
& & $\begin{array}{l}\text { thalamotomy [21] } \\
\text { Histotripsy mechanical ablation [76] }\end{array}$ \\
\hline
\end{tabular}

, preclinical; $\bullet$, clinical trials; $\mathbf{\square}$, regulatory clinical approval; MRgFUS, transcranial MR-guided focused ultrasound; BBBO, blood-brain barrier opening.

plains how large Virchow-Robin spaces, ventricular structures, and large-caliber blood vessels might act as heat sinks.

Cavitation refers to the interaction of acoustic waves with gas- or vapor-filled cavities. Preformed microbubbles are injected intravenously to facilitate stable cavitation necessary to open the BBB [33] and are available as ultrasound contrast agents. With stable or noninertial cavitation, bubbles oscillate, resulting in mechanical forces on the vessel wall thought to be mainly responsible for altering the structural and functional components of the BBB (e.g., occludin and ZO-1, caveolae, and $\mathrm{P}$-glycoprotein). The safe and effective $\mathrm{BBBO}$ in nearly every brain region has been studied in animal models and reviewed in depth previously [34]. The extent of $\mathrm{BBBO}$ is dependent on a number of factors: ultrasound parameters (e.g., peak negative pressure, burst length, burst repetition frequency, and exposure duration), microbubble dose (e.g., concentration, size distribution, and circulation time), and intrinsic tissue properties (e.g., vascular density). One commonly used commercial ultrasound contrast agent is Definity ${ }^{\circledR}$ (Lantheus Medical Imaging), which are inert gas perflutren-containing lipid microspheres with a main size distribution of $1.1-3.3 \mu \mathrm{m}$ and a half-life of in vivo approximately $1.3 \mathrm{~min}$. Capillaries in the brain have a diameter between approximately 6 and $10 \mu \mathrm{m}$ [35]. Such microspheres have similar rheology as red blood cells. Therefore, differences in vessel properties, like vascular density and fragility, can alter the result of BBBO. In summary, the therapeutic FUS can be classified by frequency, intensity, and addition of microbubbles, with different applications dependent on the dominant biologic effect (Table 1).

\section{Principles of Optimal Treatment}

There are several modifiable factors to increase patient tolerability and overall treatment success. The overarching goal is to minimize acoustic attenuation in the near field - between the transducer and the target - and maximize energy deposition at the intended focus. These factors will be discussed in chronological order of the clinical workflow (Fig. 2).

\section{Patient Selection}

\section{General}

Patients must meet disease-specific criteria for the MRgFUS. Medical contraindications for MRgFUS procedures include uncorrected coagulopathy and the American Society of Anesthesiologists physical status classification 4 or higher. Presurgical anesthetic assessments are arranged for all patients. In addition, patients should be made aware during the consent process of the need for a full head shave and placement of a stereotactic frame. Patients with mild-to-moderate claustrophobia may still be able to tolerate MRgFUS procedures on a case-by-case basis. Coagulopathies should be corrected such that the international normalized ratio is $<1.2$. Platelet count should also be above $100,000 / \mu \mathrm{L}$. Patients on anticoagulation or antiplatelet therapies can be asked to hold these medications for the procedures if the associated risks are low.

\section{Thermoablation}

Skull morphology characteristics are important determinants of temperature rise during thermal MRgFUS 


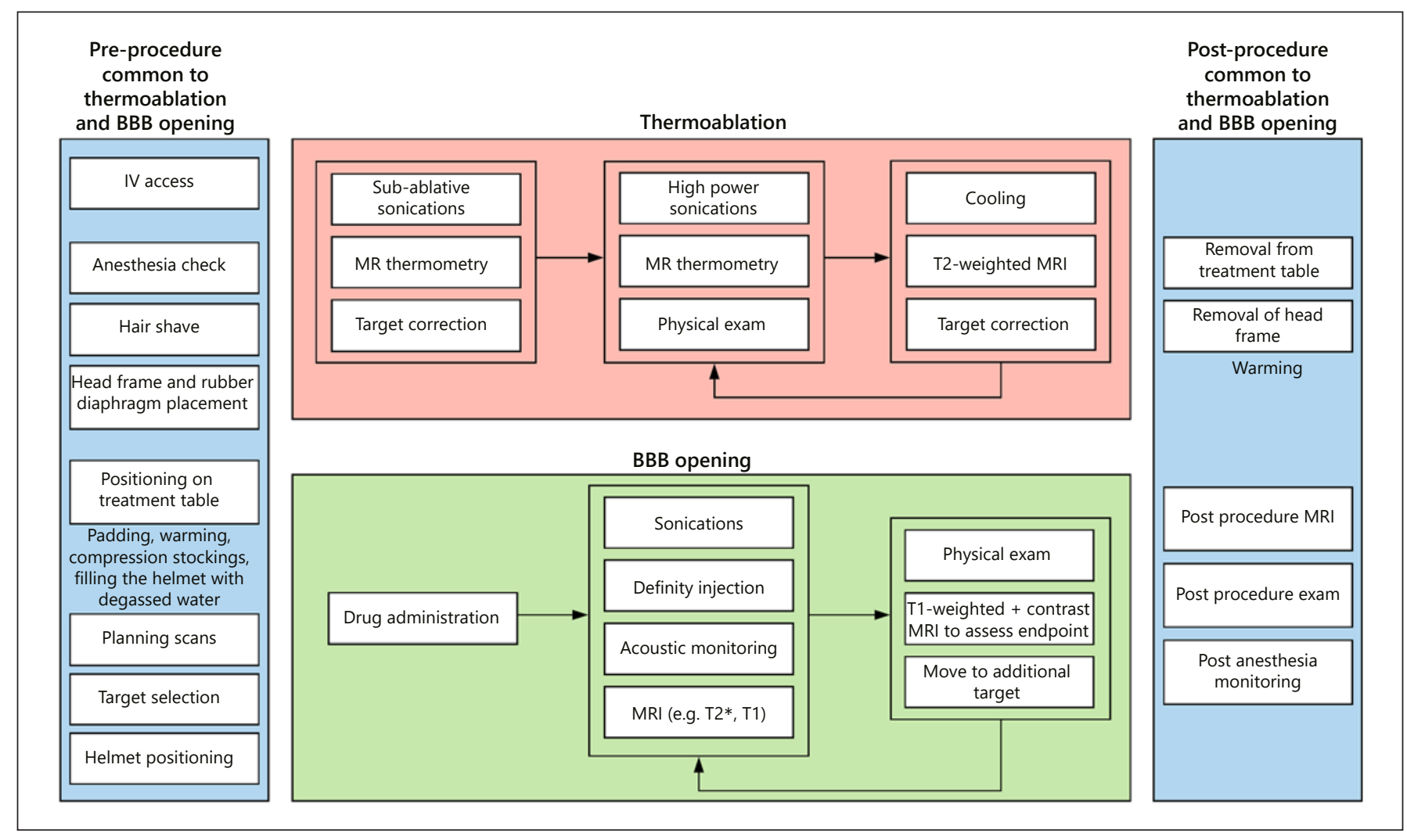

Fig. 2. Clinical workflow common to all MRgFUS brain treatments is depicted in blue (left, right), together with steps specific to thermoablation (red, middle top) and BBBO (green, middle bottom) procedures. MRgFUS, transcranial MR-guided focused ultrasound; BBBO, blood-brain barrier opening; IV, intravenous. brain treatments. The skull density ratio (SDR) is one such metric calculated by the ExAblate Neuro systems. SDR is calculated based on patient-specific CT scans of the head, specifically the ratio between the Hounsfield intensity of the trabecular and cortical bone layers averaged across the trajectories between the target and each active element in the transducer array [36]. Thus, SDR is not a fixed value for each individual and depends on the anatomical location of the target as well as the number of transducers for which an unobstructed path to the target exists. To ensure proper estimation of SDR using existing transcranial MRgFUS systems, helical CT should be acquired covering the entire head with a slice thickness of $1 \mathrm{~mm}$ or less, reconstructed with the appropriate convolution kernel depending on the CT brand (e.g., bone plus kernel for GE) and with no gantry tilt.

Patients with low SDR values are generally associated with low sonication efficiencies in lesional applications (i.e., ratio of focal temperature elevation to the applied acoustic energy) that limit the achievable focal tempera- tures and can preclude lesion formation. Intracranial calcifications, scalp lesions, and hyperostosis frontalis impair ultrasound transmission and have a higher propensity to heat. The presence of these characteristics is relative contraindication for thermoablation. These regions, along with sensitive neurovascular structures, can be blocked as "no-pass zones" using the MRgFUS system software, which turns off the number of active transducer elements to minimize energy deposition in these regions. Numerical simulations of transskull ultrasound propagation may provide the opportunity to investigate treatment feasibility preoperatively [37-39].

The proportion of patients unsuitable for MRgFUS thermoablation depends on a number of factors and changes in this quickly advancing field. Patients with SDR below 0.30 and or hyperostosis frontalis will be cautioned regarding the higher risk of lesioning failure but are not absolute excluded during the screening process. Indeed, some institutions exclude patients from MRgFUS thermoablation procedures based on SDR (e.g., SDR $<0.40-$ 
Fig. 3. Top: illustration of the current ExAblate clinical MRgFUS system. The CRW frame is typically positioned as inferiorly as possible to maximize the cranial surface area that is exposed to the transducers. Bottom: diagram illustrating the injection port for microbubbles in BBBO applications. MRgFUS, transcranial MR-guided focused ultrasound; CRW, Cosman-Roberts-Wells; BBBO, blood-brain barrier opening; IV, intravenous.

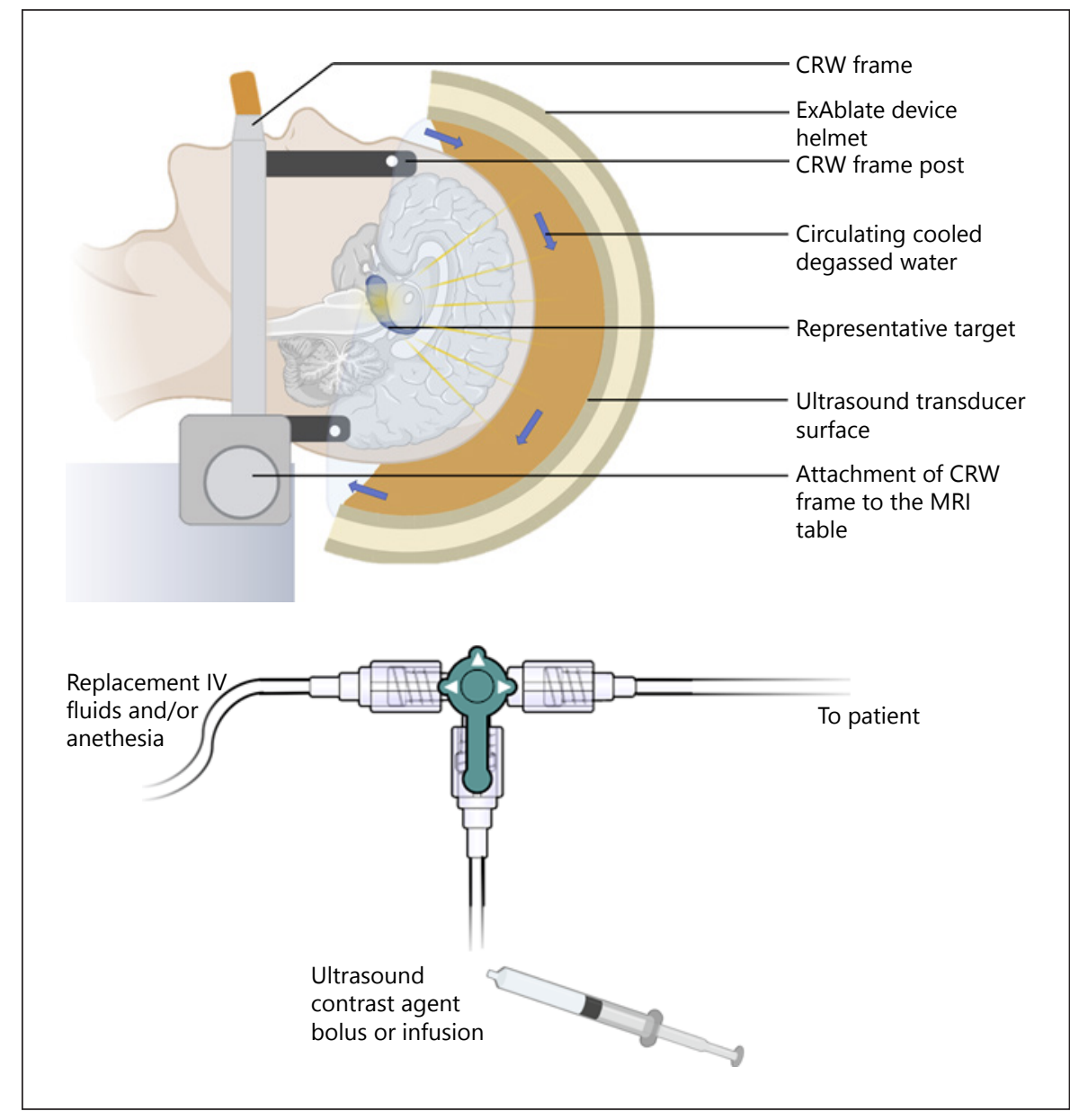

$0.45)[5,36,40]$. However, recent advancements in transcranial ultrasound focusing capabilities using the clinical device have resulted in improved sonication efficiency, which may ultimately increase the proportion of patients that are "treatable" via MRgFUS as well as enable successful lesioning in previously failed patients [41].

\section{BBB Opening}

A patient might be excluded from participation in FUS $\mathrm{BBBO}$ procedures for a variety of study criteria including disease-specific scores, contraindications to delivered therapies, MRI, MRI contrast, and ultrasound contrast agent (e.g., Definity ${ }^{\circledR}$ ). Specifically, contraindications to the latter include right-to-left shunts and severe cardiopulmonary disease among others that can be found in the product monograph.

For nonthermal microbubble-mediated MRgFUS procedures, skull properties are less critical for patient selection. First, the lower array operating frequency reduces the impact of skull-induced beam distortions [42].
Second, because the time-averaged acoustic powers required for microbubble-mediated BBB permeabilization are several orders of magnitude lower than those required for thermoablation $[11,12]$, the applied acoustic power can be increased until an appropriate level of cavitation activity is induced at the desired target without risk of skull heating. Patient tolerability of prolonged MRI sessions remains an important consideration. However, during nonthermal procedures, symptom feedback is less critical for treatment guidance, and thus patients can be more deeply sedated with careful selection of anesthetic agents.

\section{Patient Preparation}

Patient preparation for both thermal and nonthermal MRgFUS procedures follows similar protocols. Of importance is a meticulously clean shave to avoid air bubbles trapped in the hair [43]. Shaving cream may be used 
to reduce skin irritation after procedure, but should be entirely wiped away prior to patient setup. During the assemblage of the MR compatible Cosman-Roberts-Wells (CRW) stereotactic frame (Integra ${ }^{\circledR}$; UCHRA), attention should be paid to correct placement of the numbered posts. In general, placement of the stereotactic frame can be placed under local anesthetic only, and wherever possible, intravenous (IV) adjuncts should be avoided due to side effects like nausea and oversedation. Furthermore, the frame should be positioned as inferiorly as possible on the head to maximize the number of active transducer elements (Fig. 3).

In placing the frame, we generally start with the front 2 head ring screws advanced $3 / 4$ of the way in, in an attempt to avoid the temporalis muscle. We do not use a torque wrench when tightening the screws but do administer 1-2 more turns after they are finger tight. Adequate fixation of the head is particularly important for thermoablation treatments, both for precise stereotactic targeting and accurate temperature monitoring as the MR thermometry is sensitive to motion.

Attention should be given to the location of the target. Ideally, the target is placed as close to the transducer array's geometric focus as possible. The helmet can be translated and rotated mechanically with respect to the patient's head; however, when extreme targets are attempted, the range of motion can be limited physically by the stereotactic frame or MRI table. For cerebellar targets, for instance, the frame should be placed as inferior as possible. Shorter MR-compatible disposable head ring screws (Integra ${ }^{\circledR}$; DHRSS5) can be used when a significant helmet tilt is necessary to provide maximal clearance, provided the subject head size permits. Finally, expanders or "ear" adapters are available to offset the frame even more from the helmet. A final check of any hyperflexion or hyperextension of the neck when the frame is parallel to the floor is important, particularly for patients with pre-existing cervical spine disease.

\section{Intraoperative}

\section{Thermoablation}

The clinical protocol for MRgFUS thermoablation and lesion prediction has been described previously [44-46]. Accurate location of the temperature elevation on axial and sagittal MR thermometry with sublesional sonications is required. MRI monitoring during sonications is an important feature of MRgFUS. Planning scans are acquired as described previously. Intraoperative thermom-

Transcranial MR-Guided Focused Ultrasound etry is critical both for spatial accuracy and estimation of accumulated thermal dose, relevant to guiding treatment endpoints $[30,31]$. Targeting accuracy of the thermal lesion can be tested with online MR thermometry in 3 orientations during subablative temperature sonications. Subsequent corrections can be made depending on the orientation and discrepancy if needed. In the MR thermometry employed by the ExAblate Neuro system, temperature changes are measured based on phase differences between dynamic frames in a single $2 \mathrm{D}$ plane. These differences are linearly related to temperature change in relevant ranges but are sensitive to motion. T2-weighted imaging is also helpful with regard to guiding treatment for instance to confirm lesion formation and assess size and location [47].

Bouts lasting $40-50 \mathrm{~s}$ of sequentially higher-power FUS in the range of 500-900 W are then delivered alternating with clinical examinations. Clinical examinations assess for treatment effects and adverse events that might advise spatial adjustment of the target. In general, we aim for $2-3$ bouts of temperature elevation above $54^{\circ} \mathrm{C}$. A T2 sequence is performed after a group of sonications between 50 and $57^{\circ} \mathrm{C}$ or when the patient experiences good clinical improvement to assess treatment endpoint. We aim for a lesion measuring $4-5 \mathrm{~mm}$ in diameter on the intraoperative $\mathrm{T} 2$-weighted sequence. The lesion size 1 day after the procedure is expected to expand by $1-2 \mathrm{~mm}$. Lesion size can be predicted by the accumulated thermal dose model, which accounts for additional factors such as heat diffusion to adjacent tissue and oblique focus [30, 31, 48].

Overall, the total time on the MRgFUS system for thermoablation ranges from 2 to $3 \mathrm{~h}$. In terms of anesthesia, sedation and analgesia are critically important during thermal FUS procedures for scalp heating and pain [49]. Scalp pain from high-power FUS is transient and does not typically require further treatment postoperatively. Acetaminophen is a typically effective analgesic for mild postoperative pain associated with cold water or stereotactic frame. A few hours of observation and supportive care is typically sufficient prior to patient being ready for discharge.

\section{BBB Opening}

For BBBO procedures, a three-way stopcock is added on the IV line such that it is accessible for the ultrasound contrast agent injections while the patient is in the MRI bore (Fig. 3). Microbubble contrast agent administration can be carried out using repeated boluses or using a continuous infusion. For bolus administrations of Definity ${ }^{\circledR}$ 


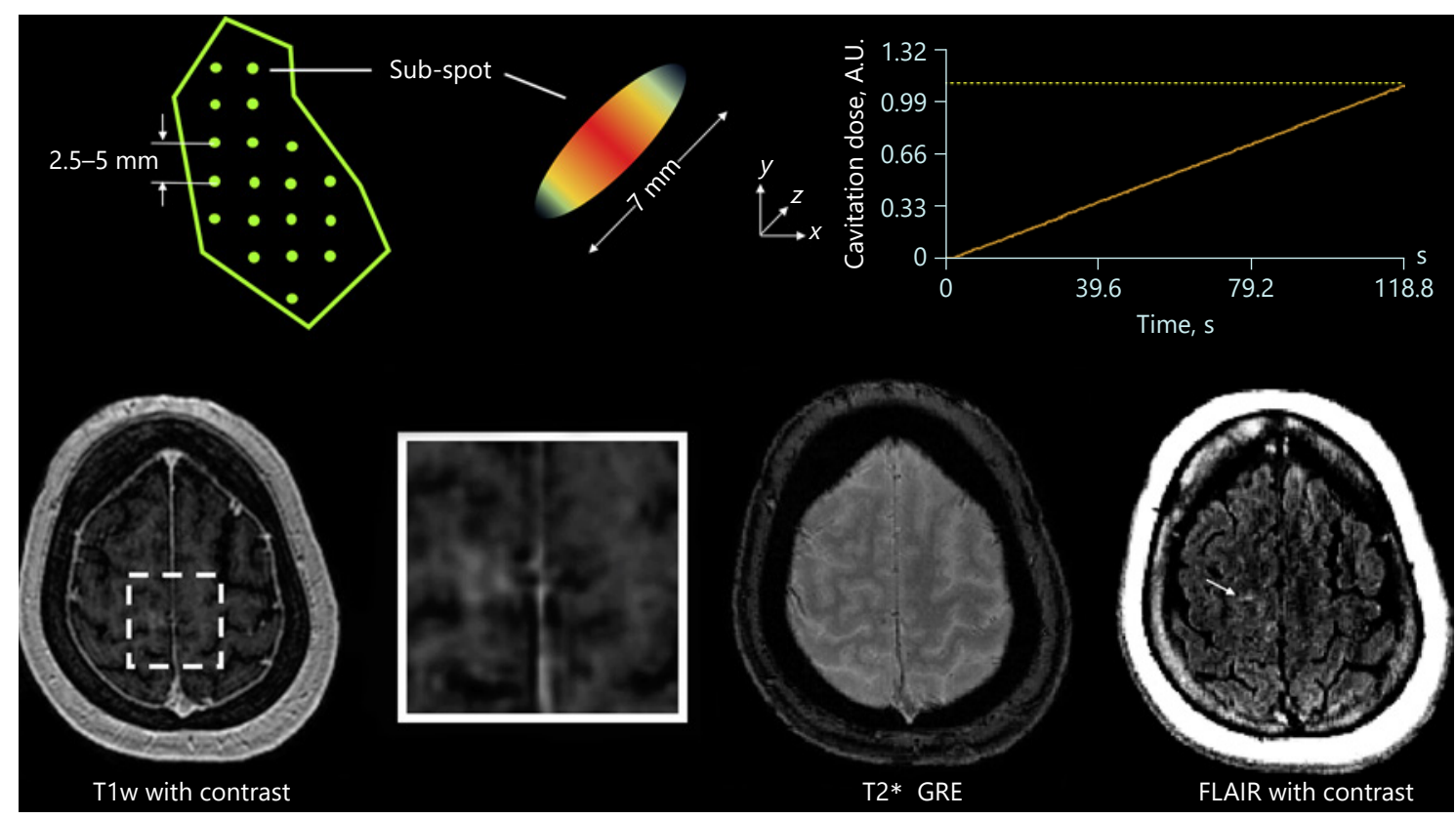

Fig. 4. Top: schematic illustration of planning and system feedback for MRgFUS BBBO procedures. The current ExAblate system software provides freehand or polygon tools for creation of the TV and automatically fills in the spots (top left). Spot spacing and number of spots are customizable, within the limits of achieving the desired pulsing protocol. Each spot has a depth of approximately $7 \mathrm{~mm}$ (top middle). Every spot within the target receives the same transmitted ultrasound power, but the resultant cavitation dose can be heterogeneous. The cavitation dose data are measured by acoustic emissions and can be visually represented at the treatment console spatially or over time (top right). Bottom: contrast-enhanced T1weighted and FLAIR sequences are useful in assessing FUS-induced bioeffects during BBBO procedures. Other sequences including T2* GRE and DWI collectively help evaluate adverse events such as bleeding and ischemia. MRgFUS, transcranial MRguided focused ultrasound; BBBO, blood-brain barrier opening; $\mathrm{TV}$, treatment volume; FLAIR, fluid-attenuated inversion recovery; GRE, gradient echo; DWI, diffusion weighted imaging. as an example, a $4 \mu \mathrm{L} / \mathrm{kg}$ dose is injected $30-40$ s prior to the start of sonication followed by a 5 - to 10 -cc saline flush enough to clear the microbubbles from the IV line [33]. The half-life of Definity ${ }^{\circledR}$ is $1.3 \mathrm{~min}$, allowing approximately $5 \mathrm{~min}$ of effective sonication, and must therefore be redosed every $5 \mathrm{~min}$. While the maximum indicated dose of Definity ${ }^{\circledR}$ for diagnostic imaging is $20 \mu \mathrm{L} /$ $\mathrm{kg}$, up to $100 \mu \mathrm{L} / \mathrm{kg}$ has been tested in initial clinical safety testing. Early MRgFUS trials adhered to $20 \mu \mathrm{L} / \mathrm{kg}$, which was expanded following accumulation of safety data. For infusion administrations, $1.3 \mathrm{cc}$ of Definity ${ }^{\circledR}$ is mixed into a 500 -cc preservative-free saline bag and infused at $10 \mathrm{cc} / \mathrm{min}$. Infusion allows this aspect of the procedure to be operator independent and allows continuous sonications, saving time and frequent interactions with the IV line. The potential disadvantages of infusion include settling of microbubbles over time, agent waste, and a lower temporal-peak microbubble concentration compared to bolus administrations. Finally, administration of $\mathrm{O}_{2}$ has been shown to reduce the effectiveness of $\mathrm{BBBO}$ in animals [50], potentially due to decreased concentration of microbubbles in cerebral circulation from vasoconstriction [51]. Therefore, ideally, patients are removed of supplementary oxygen during sonications if safe to do so.

Because microbubble-mediated FUS applications are affected by the concentration and size distribution of microbubbles, operators should also be well versed with appropriate handling of Definity ${ }^{\circledR}$ and other similar ultrasound contrast agents [52]. Definity ${ }^{\circledR}$ is stored long term at $2-8^{\circ} \mathrm{C}$ and does not contain bacterial preservative. The vial should be allowed to reach room temperature $(\sim 20$ min) prior to activation, as activation temperature has been found to affect the resulting size distribution [53]. Activation involves mixing in the VIALMIX ${ }^{\circledR}$ apparatus (Lantheus Medical Imaging) for $45 \mathrm{~s}$ immediately before use, whereby the liquid turns from clear to milky. The microbubbles settle with time, so should be resuspended with repeated inversions of the vial or syringe for $10 \mathrm{~s}$. Only 18- to $20-\mathrm{G}$ needles should be used to draw up the 
Definity ${ }^{\circledR}$, without injecting air into the vial. Previous work has shown rapid injection with small needles can damage the microbubbles, resulting in considerable differences in concentration and size distribution [54].

During planning stages, target regions are hand drawn over the planning MRI. For BBBO, polygon tools can be used to place a treatment volume (TV) with spacing of sonication spots predefined (Fig. 4). Smaller grid spacings (e.g., $2.5 \mathrm{~mm}$ ) are preferred for more homogeneous BBBO and smaller anatomic structures. Each subspot has a focal depth of approximately $7 \mathrm{~mm}$, which is about the full width at half maximum along the $230-\mathrm{kHz}$ ultrasound beam [19].

Each TV consists of 1 bout of sonication and estimates the overall cavitation dose delivered. The maximum number of spots for TV - approximately 30 - takes into account the distance between spots and ultrasound parameters (e.g., duty cycle and repetition frequency) that are permissible via electronic steering of the transducers. However, larger volumes or more spots often result in greater heterogeneity of cavitation dose, and any spot in the TV may terminate the sonication if a dose threshold is reached. Currently, the power is not modulated by spot acoustic feedback.

For BBBO, MR thermometry is less useful as previous studies have shown macroscopic temperature changes are negligible $[11,12] . \mathrm{T} 2 *$-weighted gradient echo and susceptibility-weighted imaging sequences are useful to evaluate for microhemorrhages. Additionally, acoustic emission data can be used to predict the induced biological effects [55, 56]. T1-weighted MRI after gadolinium injection can visualize changes in BBB permeability and help determine treatment endpoints. However, once gadolinium is administered, it remains in the brain for the duration of the procedure. Thus, contrast-enhanced MRI lacks fidelity as a continuous measure of the effect of ultrasound.

Alternatively, actively controlled FUS exposure using acoustic-based monitoring is an emerging strategy to automate safe and effective procedures [57-59]. The detection of acoustic signals by passive cavitation detectors within the helmet and real-time decomposition into a frequency spectrum can be used to assess characteristics of the microbubble activity in situ [60]. Further, the use of multielement hemispherical detector arrays allows threedimensional spatial localization of the induced microbubble activity within the brain [61], which has been demonstrated in several preclinical studies $[19,62]$. This approach has recently shown utility in predicting FUS bioeffect volume distributions [63]. Acoustic emission

Transcranial MR-Guided Focused Ultrasound analysis offers high fidelity monitoring of the BBBO process, promising to render the procedure safer and more efficient and real-time MRI unnecessary. Work is ongoing to predict the quality of $\mathrm{BBBO}$ with the cavitation dose detected from the calibration of cavitation detectors on the current low-intensity MRgFUS clinical prototype.

There is no scalp pain during FUS BBBO procedures, rather participants complain of coldness from subroom temperature water circulation. Because $\mathrm{BBBO}$ procedures may be prolonged depending on the total TV, up to $4 \mathrm{~h}$ within the device, participants are prone to becoming anxious or bored due to long periods of immobility. Other participants develop claustrophobia over repeated procedures. We find dexmedetomidine infusion to be helpful in maintaining patient cooperation and comfort during these long procedures. Other anesthetic protocols used for awake craniotomies might also be useful. Warming blanket or other devices are helpful in improving the tolerability of the procedure and postoperative recovery.

Similar to thermoablation, postoperative recovery requirements are minimal. Postoperative physical exams are conducted to document adverse events such as those related to FUS or the procedure. Patients might require a brief period of recovery with supportive care (e.g., nonopioid analgesia and antiemetics) prior to being ready for discharge.

\section{Radiologic Results}

In general, structural T1- and T2-weighted sequences are acquired to assess size and morphology of lesions. These characteristics, along with spatial location, have been shown to be correlated with clinical outcomes as well as adverse events $[64,65]$. Time course of lesion development involves expansion of the lesion in the initial $24 \mathrm{~h}$, stabilization, and potential regression over the course of months $[66,67]$. Early postoperative susceptibility-weighted imaging has been found to be predictive of lesion appearance at 6 months [66].

For BBBO procedures, MRI contrast agent extravasation on MRI can be used to measure BBB permeability and taken as a surrogate for drug delivery [68]. For instance, Gadovist ${ }^{\circledR}$ is approximately $600 \mathrm{Da}$ and does not cross the intact BBB. The appearance of enhancement within the sonicated target can appear well demarcated, such as reported in a group of patients with $\mathrm{AD}$ [12], or heterogeneous and "fluffy" as found in a group of patients with glioblastoma [13], possibly depending on underlying tissue properties (e.g., interstitial pressure, grey vs. 
white matter, and vascular density). Blood-pool gadolinium-based contrast agents (e.g., gadofosveset) that bind to albumin $(66 \mathrm{kDa})$ have been shown to enhance sonicated targets following BBBO as well but at substantially lower levels than nonblood pool agents [69]. Gadofosveset provides lower sensitivity of BBBO but may provide greater validity as a surrogate marker for enhanced delivery of larger molecular drugs.

The presence of MRI contrast agent within the subarachnoid and perivascular spaces appears highly intense on fluid-attenuated inversion recovery (FLAIR) sequences, which also has a differential diagnosis of acute subarachnoid hemorrhage or meningitis [70]. Contrast enhancement on FLAIR after BBBO allows visualization of gadolinium clearance through glymphatic pathways and resolves once $\mathrm{BBB}$ permeability is restored [71]. In addition, T2, T2* gradient echo, and diffusion weighted imaging are useful for assessing adverse events following sonications, such as swelling, bleeding, and ischemia. In our experience, post-BBBO T2* changes tend to resolve on follow-up studies the day after the procedure [12]. Functional imaging, diffusion tensor imaging, and arterial spin labeling techniques are amongst the array of research MR sequences available to further characterize the biological effects of FUS-mediated increases in BBB permeability $[72,73]$.

Following MRgFUS procedures, participants are admitted to the hospital for observation and often can meet standard criteria for discharge within a few hours. For thermoablation, the follow-up MRI 1 day after treatment provides a more accurate assessment of lesion size. A delayed clinical assessment here is important given new adverse events. For BBBO cases, MRI evaluation 1 day after treatment is useful for assessing restoration of BBB permeability and evolution of any signal change on other MR sequences.

\section{Challenges and Future Directions}

Existing challenges in MRgFUS thermoablation include expanding the treatment envelope, increasing the number of eligible candidates for MRgFUS, streamlining the procedure workflow, and incorporating more patient-centered outcome measures. The treatment envelope refers to the volume of brain tissue within the skull cavity that can be targeted and treated successfully. With existing MRgFUS technology, sonication efficiency is higher for central targets (e.g., thalamotomy) than for peripheral targets (e.g., capsulotomy) and is higher in some patients than others [74]. One approach to improve sonication efficiency is to employ transskull ultrasound focusing based on acoustic measurements collected during treatment $[75,76]$. A similar approach has been demonstrated to be successful and safe in patients with ET using the high-frequency ExAblate Neuro system [41]. In terms of retreatment or bilateral treatments, case series of patients with obsessive-compulsive disorder and major depressive disorder have demonstrated that bilateral MRgFUS capsulotomy is feasible [77, 78]. Clinical testing of bilateral staged thalamotomy and subthalamotomy is ongoing (NCT04501484, NCT03465761, and NCT03964272). Data from early MRgFUS thalamotomy cases show sonication efficiency can decrease over time within a single session potentially caused by changes in acoustic properties of the skull and brain tissue due to heating [48]. This limitation will have implication on the feasibility of bilateral procedures conducted in a single session. In recent years, there has also been an increased engagement of the entire health care team in understanding the unique implications of this novel technology $[49,79]$.

The treatment envelope within the brain is substantially expanded for BBBO procedures, allowing, for instance, treatment of the hippocampus and medial temporal lobe [15]. However, it is still our experience that treating extreme locations within the skull (e.g., the base of the anterior middle fossa, occipital pole, and lateral cerebellum) is more challenging using MRgFUS brain systems. This is largely due to the difficulty of placing the stereotactic frame such that the helmet's geometric center is as close as possible to the target. In this regard, a freely moving FUS array could offer greater flexibility in targeting. The development of a frameless system, such as, for example, with the NaviFUS system, where targeting is based on preoperative MRI, may facilitate this. Furthermore, frameless systems are generally better tolerated by patients and reduce procedure time. However, the greater flexibility comes with a tradeoff of a decrease in precision. The spatial error of frameless systems is at least 2 times greater than frame-based systems [80].

Automation of ultrasound exposure delivery and monitoring to accurately predict treatment endpoint will enable larger tissue volume to be treated in a shorter amount of time. Acoustic emission patterns from ultrasound-stimulated microbubbles have been shown to predict successful BBBO $[58,59,81]$. The same signals have been shown to correlate with the level of increase in BBB permeability, as measured permeability coefficient Ktrans from dynamic contrast-enhanced MRI [82]. This relationship, however, has yet to be studied in 
human subjects. Acoustic feedback, compared to feedback by contrast-enhanced T1w MRI, offers higher fidelity during continuous monitoring since gadolinium has a dose limitation and half-life. On the other hand, T2* acquisition during sonications can be useful for early detection of adverse events. Nevertheless, the signalto-noise ratio of intraoperative MRI acquisitions still desires improvement. In early clinical MRgFUS BBBO studies, intraoperative MR thermometry confirmed that focal temperature elevations are negligible during the low duty-cycle exposures employed for this application [12].

Finally, the practicalities of combining FUS with a drug and the effect of FUS on its pharmacokinetic profile still have many unanswered questions. For example, what is the optimal time to inject the drug? Dynamic microscopy studies in animals show fast and slow leakage of intravenously injected tracer after FUS BBBO and peak increase in permeability within $5 \mathrm{~min}$ of sonications $[83,84]$. Notably, fast leakage appeared to dominate with higher acoustic pressures and larger caliber $(30-70 \mu \mathrm{m})$ vessels [84]. Together, these results suggest the drug should ideally reach peak plasma concentration during sonications. Will it be feasible to combine FUS to therapies with dosing regimen as frequently as weekly? By how much does FUS improve the tissue concentration of a drug and how should the systemic dose be adjusted as a result, if at all? Several animal studies have looked at this for specific drugs or more generally using Ktrans in different tissue including tumor $[85,86]$. Another approach to addressing this question in vivo is through radiopharmaceuticals, which has been investigated in animal models [87]. However, radiopharmaceuticals are specific to each drug and costly and time consuming to develop.

\section{Conclusion}

This review provides an overview of how to optimize clinical research protocols for transcranial applications of MRgFUS, with an emphasis on microbubble-mediated BBBO procedures. Continued understanding of the associated technical parameters, together with further advances in MRgFUS technology, will help standardize and optimize treatment procedures, as well as expand indications in the future.

\section{Statement of Ethics}

Not applicable.

\section{Conflict of Interest Statement}

N.L. has received honorarium for serving on an expert steering committee for the Focused Ultrasound Foundation, as well as research support from the Focused Ultrasound Foundation and InSightec. K.H. is an inventor of intellectual property related to the brain application of focused ultrasound owned by the Brigham and Women's Hospital and Sunnybrook Research Institute. Other authors have no potential conflicts of interest to disclose.

\section{Funding Sources}

Y.M. was supported by the research fellowships from the Natural Sciences and Engineering Research Council, Physicians' Services Incorporated, and Hold'em for Life Foundations.

\section{Author Contributions}

Y.M., R.M.J., B.D., Y.H. C.B.P., and S.S. were all involved in data collection, writing, and editing of the manuscript. C.H., N.L., and K.H. critically reviewed and approved the manuscript.

\section{References}

1 Focused Ultrasound Foundation. 2019 State of the Field Report. 2018. Available from: http: //www.fusfoundation.org/images/pdf/ FUSF_State_of_the_Field_Report_2019.pdf.

2 Alexandrov AV, Köhrmann M, Soinne L, Tsivgoulis G, Barreto AD, Demchuk AM, et al. Safety and efficacy of sonothrombolysis for acute ischaemic stroke: a multicentre, double-blind, phase 3, randomised controlled trial. Lancet Neurol. 2019 Apr;18(4): $338-47$.

3 Lee W, Kim HC, Jung Y, Chung YA, Song IU, Lee JH, et al. Transcranial focused ultrasound stimulation of human primary visual cortex. Sci Rep. 2016 Sep;6:34026.
4 Bond AE, Shah BB, Huss DS, Dallapiazza RF, Warren A, Harrison MB, et al. Safety and efficacy of focused ultrasound thalamotomy for patients with medication-refractory, tremor-dominant Parkinson disease: a randomized clinical trial. JAMA Neurol. 2017 Oct;74(12):1412-8.

5 Elias WJ, Lipsman N, Ondo WG, Ghanouni P, Kim YG, Lee W, et al. A randomized trial of focused ultrasound thalamotomy for essential tremor. N Engl J Med. 2016 Aug; 375(8):730-9.
6 Lipsman N, Schwartz ML, Huang Y, Lee L, Sankar T, Chapman M, et al. MR-guided focused ultrasound thalamotomy for essential tremor: a proof-of-concept study. Lancet Neurol. 2013 May;12(5):462-8.

7 Li C, Gajic-Veljanoski O, Schaink AK, Higgins $\mathrm{C}$, Fasano A, Sikich N, et al. Cost-effectiveness of magnetic resonance-guided focused ultrasound for essential tremor. Mov Disord. 2019 May;34(5):735-43.

8 Ravikumar VK, Parker JJ, Hornbeck TS, Santini VE, Pauly KB, Wintermark M, et al. Cost-effectiveness of focused ultrasound, radiosurgery, and DBS for essential tremor. Mov Disord. 2017 Apr;32(8):1165.
Transcranial MR-Guided Focused Ultrasound
Stereotact Funct Neurosurg 2021;99:329-342 DOI: $10.1159 / 000512111$ 
9 Abrahao A, Meng Y, Llinas M, Huang Y, Hamani C, Mainprize T, et al. First-in-human trial of blood-brain barrier opening in amyotrophic lateral sclerosis using MRguided focused ultrasound. Nat Commun. 2019 Sep;10(1):4373-9.

10 Carpentier A, Canney M, Vignot A, Reina V, Beccaria K, Horodyckid C, et al. Clinical trial of blood-brain barrier disruption by pulsed ultrasound. Sci Transl Med. 2016 Jun;8(343):343re2.

11 Hynynen K, McDannold N, Vykhodtseva N, Jolesz FA. Noninvasive MR imaging-guided focal opening of the blood-brain barrier in rabbits. Radiology. 2001 Sep;220(3):640-6.

12 Lipsman N, Meng Y, Bethune AJ, Huang Y, Lam B, Masellis M, et al. Blood-brain barrier opening in Alzheimer's disease using MRguided focused ultrasound. Nat Commun. 2018 Jul;9(1):2336.

13 Mainprize T, Lipsman N, Huang Y, Meng Y, Bethune A, Ironside S, et al. Blood-brain barrier opening in primary brain tumors with non-invasive MR-guided focused ultrasound: a clinical safety and feasibility study. Sci Rep. 2019 Jan;9(1):321.

14 Park SH, Kim MJ, Jung HH, Chang WS, Choi HS, Rachmilevitch I, et al. Safety and feasibility of multiple blood-brain barrier disruptions for the treatment of glioblastoma in patients undergoing standard adjuvant chemotherapy. J Neurosurg. 2020 Jan; 1(aop):1-9.

15 Rezai AR, Ranjan M, D'Haese P-F, Haut MW, Carpenter J, Najib U, et al. Noninvasive hippocampal blood-brain barrier opening in Alzheimer's disease with focused ultrasound. Proc Natl Acad Sci U S A. 2020 Apr;117(17):9180-2.

16 Marchi N, Angelov L, Masaryk T, Fazio V, Granata T, Hernandez N, et al. Seizure-promoting effect of blood-brain barrier disruption. Epilepsia. 2007;48(4):732-42.

17 Sun T, Dasgupta A, Zhao Z, Nurunnabi M, Mitragotri S. Physical triggering strategies for drug delivery. Adv Drug Deliv Rev. 2020 Jun;S0169409X20300569.

18 Asquier N, Bouchoux G, Canney M, Martin C, Law-Ye B, Leclercq D, et al. Blood-brain barrier disruption in humans using an implantable ultrasound device: quantification with MR images and correlation with local acoustic pressure. J Neurosurg. 2019 Feb; 132(3):875-83.

19 Jones RM, Deng L, Leung K, McMahon D, O’Reilly MA, Hynynen K. Three-dimensional transcranial microbubble imaging for guiding volumetric ultrasound-mediated blood-brain barrier opening. Theranostics. 2018;8(11):2909-26.

20 Liu H-L, Tsai C-H, Jan C-K, Chang H-Y, Huang S-M, Li M-L, et al. Design and implementation of a transmit/receive ultrasound phased array for brain applications. IEEE Trans Ultrason Ferroelectr Freq Control. 2018 Oct;65(10):1756-67.
21 Pouliopoulos AN, Wu SY, Burgess MT, Karakatsani ME, Kamimura HAS, Konofagou EE. A clinical system for non-invasive blood-brain barrier opening using a neuronavigation-guided single-element focused ultrasound transducer. Ultrasound Med Biol. 2020 Jan;46(1):73-89.

22 Clement GT, Hynynen K. A non-invasive method for focusing ultrasound through the human skull. Phys Med Biol. 2002 Apr; 47(8):1219-36.

23 McDannold N, Clement GT, Black P, Jolesz F, Hynynen K. Transcranial magnetic resonance imaging-guided focused ultrasound surgery of brain tumors: initial findings in 3 patients. Neurosurgery. 2010 Feb; 66(2): 323-32.

24 White J, Clement GT, Hynynen K. Transcranial ultrasound focus reconstruction with phase and amplitude correction. IEEE Trans Ultrason Ferroelectr Freq Control. 2005 Sep;52(9):1518-22.

25 Connor CW, Hynynen K. Patterns of thermal deposition in the skull during transcranial focused ultrasound surgery. IEEE Trans Biomed Eng. 2004 Oct;51(10):1693-706.

26 Fry FJ, Barger JE. Acoustical properties of the human skull. J Acoust Soc Am. 1978 May;63(5):1576-90.

27 Sapareto SA, Dewey WC. Thermal dose determination in cancer therapy. Int J Radiat Oncol Biol Phys. 1984 Jun;10(6):787-800.

28 Roizin-Towle L, Pirro JP. The response of human and rodent cells to hyperthermia. Int J Radiat Oncol Biol Phys. 1991 Apr;20(4): 751-6.

29 Dewhirst MW, Viglianti BL, Lora-Michiels M, Hanson M, Hoopes PJ. Basic principles of thermal dosimetry and thermal thresholds for tissue damage from hyperthermia. Int J Hyperthermia. 2003 Jun;19(3):267-94.

30 Huang Y, Lipsman N, Schwartz ML, Krishna V, Sammartino F, Lozano AM, et al. Predicting lesion size by accumulated thermal dose in MR-guided focused ultrasound for essential tremor. Med Phys. 2018 Oct;45(10): 4704-10.

31 Jones RM, Kamps S, Huang Y, Scantlebury N, Lipsman N, Schwartz ML, et al. Accumulated thermal dose in MRI-guided focused ultrasound for essential tremor: repeated sonications with low focal temperatures. J Neurosurg. 2019 May;1-8.

32 Pennes HH. Analysis of tissue and arterial blood temperatures in the resting human forearm. J Appl Physiol. 1948 Aug;1(2):93122.

33 Huang Y, Alkins R, Schwartz ML, Hynynen $\mathrm{K}$. Opening the blood-brain barrier with $\mathrm{MR}$ imaging-guided focused ultrasound: preclinical testing on a trans-human skull porcine model. Radiology. 2017 Jan;282(1): 123-30.
34 Meng Y, Pople CB, Lea-Banks H, Abrahao A, Davidson B, Suppiah S, et al. Safety and efficacy of focused ultrasound induced blood-brain barrier opening, an integrative review of animal and human studies. J Control Release. 2019 Sep;309:25-36.

35 Atkinson JL, Anderson RE, Sundt TM. The effect of carbon dioxide on the diameter of brain capillaries. Brain Res. 1990 May; 517(1-2):333-40.

36 Chang WS, Jung HH, Zadicario E, Rachmilevitch I, Tlusty T, Vitek S, et al. Factors associated with successful magnetic resonance-guided focused ultrasound treatment: efficiency of acoustic energy delivery through the skull. J Neurosurg. 2016 Feb; 124(2):411-6.

37 McDannold N, White PJ, Cosgrove R. Elementwise approach for simulating transcranial MRI-guided focused ultrasound thermal ablation. Phys Rev Res. 2019 Dec;1(3): 033205.

38 Pulkkinen A, Werner B, Martin E, Hynynen K. Numerical simulations of clinical focused ultrasound functional neurosurgery. Phys Med Biol. 2014 Apr;59(7):1679-700.

39 Sammartino F, Beam DW, Snell J, Krishna V. Kranion, an open-source environment for planning transcranial focused ultrasound surgery: technical note. J Neurosurg. 2019 Mar; $1-7$.

40 Boutet A, Gwun D, Gramer R, Ranjan M, Elias GJB, Tilden D, et al. The relevance of skull density ratio in selecting candidates for transcranial MR-guided focused ultrasound. J Neurosurg. 2019 May;1-7.

41 Jones RM, Huang Y, Meng Y, Scantlebury N, Schwartz ML, Lipsman N, et al. Echo-focusing in transcranial focused ultrasound thalamotomy for essential tremor: a feasibility study. Mov Disord. 2020 Aug.

42 Yin X, Hynynen K. A numerical study of transcranial focused ultrasound beam propagation at low frequency. Phys Med Biol. 2005 Apr;50(8):1821-36.

43 Eames MD, Hananel A, Snell JW, Kassell NF, Aubry JF. Trans-cranial focused ultrasound without hair shaving: feasibility study in an ex vivo cadaver model. J Ther Ultrasound. 2013;1(1):24.

44 Meng Y, Huang Y, Solomon B, Hynynen K, Scantlebury N, Schwartz ML, et al. MRIguided focused ultrasound thalamotomy for patients with medically-refractory essential tremor. J Vis Exp. 2017 Dec;(130):56365.

45 Ghanouni P, Pauly KB, Elias WJ, Henderson J, Sheehan J, Monteith S, et al. Transcranial MRI-guided focused ultrasound: a review of the technologic and neurologic applications. AJR Am J Roentgenol. 2015 Jul; 205(1):150-9.

46 Davidson B, Hamani C, Huang Y, Jones RM, Meng Y, Giacobbe P, et al. Magnetic resonance-guided focused ultrasound capsulotomy for treatment-resistant psychiatric disorders. Oper Neurosurg. 2020 Jul;opaa240. 
47 Gagliardo C, Cannella R, Quarrella C, D’Amelio M, Napoli A, Bartolotta TV, et al. Intraoperative imaging findings in transcranial MR imaging-guided focused ultrasound treatment at $1.5 \mathrm{~T}$ may accurately detect typical lesional findings correlated with sonication parameters. Eur Radiol. 2020 Apr; 30(9):5059-70.

48 Hughes A, Huang Y, Schwartz ML, Hynynen $\mathrm{K}$. The reduction in treatment efficiency at high acoustic powers during MR-guided transcranial focused ultrasound thalamotomy for essential tremor. Med Phys. $2018 \mathrm{Jul}$; 45(7):2925-36.

49 Chapman M, Park A, Schwartz M, Tarshis J. Anesthesia considerations of magnetic resonance imaging-guided focused ultrasound thalamotomy for essential tremor: a case series. Can J Anesth. 2020 Apr;67(7):877-84.

50 McDannold N, Zhang Y, Vykhodtseva N. The effects of oxygen on ultrasound-induced blood-brain barrier disruption in mice. Ultrasound Med Biol. 2017 Feb;43(2): 469-75.

51 Itani M, Mattrey RF. The effect of inhaled gases on ultrasound contrast agent longevity in vivo. Mol Imaging Biol. 2012 Feb;14(1): $40-6$.

52 Goertz DE, de Jong N, van der Steen AF. Attenuation and size distribution measurements of Definity and manipulated Definity populations. Ultrasound Med Biol. 2007 Sep;33(9):1376-88.

53 Helfield BL, Huo X, Williams R, Goertz DE. The effect of preactivation vial temperature on the acoustic properties of Definity ${ }^{\mathrm{Tm}}$. Ultrasound Med Biol. 2012 Jul;38(7):1298305.

54 Talu E, Powell RL, Longo ML, Dayton PA. Needle size and injection rate impact microbubble contrast agent population. Ultrasound Med Biol. 2008 Jul;34(7):1182-5.

55 McDannold N, Vykhodtseva N, Hynynen K. Targeted disruption of the blood-brain barrier with focused ultrasound: association with cavitation activity. Phys Med Biol. 2006 Feb;51(4):793-807.

56 Tung YS, Vlachos F, Choi JJ, Deffieux T, Selert $\mathrm{K}$, Konofagou EE. In vivo transcranial cavitation threshold detection during ultrasound-induced blood-brain barrier opening in mice. Phys Med Biol. 2010 Oct;55(20): 6141-55.

57 McDannold N, Zhang Y, Supko JG, Power C, Sun T, Peng C, et al. Acoustic feedback enables safe and reliable carboplatin delivery across the blood-brain barrier with a clinical focused ultrasound system and improves survival in a rat glioma model. Theranostics. 2019;9(21):6284-99.

58 O’Reilly MA, Hynynen K. Blood-brain barrier: real-time feedback-controlled focused ultrasound disruption by using an acoustic emissions-based controller. Radiology. 2012 Apr;263(1):96-106.
59 Sun T, Zhang Y, Power C, Alexander PM, Sutton JT, Aryal M, et al. Closed-loop control of targeted ultrasound drug delivery across the blood-brain/tumor barriers in a rat glioma model. Proc Natl Acad Sci U S A. 2017 Nov;114(48):E10281-90.

60 Jones RM, Hynynen K. Advances in acoustic monitoring and control of focused ultrasound-mediated increases in blood-brain barrier permeability. Br J Radiol. 2019 Apr; 92(1096):20180601.

61 Jones RM, O'Reilly MA, Hynynen K. Transcranial passive acoustic mapping with hemispherical sparse arrays using CT-based skull-specific aberration corrections: a simulation study. Phys Med Biol. 2013 Jul; 58(14):4981-5005.

62 O’Reilly MA, Jones RM, Hynynen K. Threedimensional transcranial ultrasound imaging of microbubble clouds using a sparse hemispherical array. IEEE Trans Biomed Eng. 2014 Apr;61(4):1285-94.

63 Jones RM, McMahon D, Hynynen K. Ultrafast three-dimensional microbubble imaging in vivo predicts tissue damage volume distributions during nonthermal brain ablation. Theranostics. 2020;10(16):7211-30.

64 Meng Y, Solomon B, Boutet A, Llinas M, Scantlebury N, Huang Y, et al. Magnetic resonance-guided focused ultrasound thalamotomy for treatment of essential tremor: a 2-year outcome study: MRgFUS thalamotomy for ET: 2-year outcome. Mov Disord. 2018 Oct;33(10):1647-50.

65 Pineda-Pardo JA, Urso D, Martínez-Fernández R, Rodríguez-Rojas R, del-Alamo M, Millar Vernetti P, et al. Transcranial magnetic resonance-guided focused ultrasound thalamotomy in essential tremor: a comprehensive lesion characterization. Neurosurgery. 2019 Oct;nyz395.

66 Keil VC, Borger V, Purrer V, Groetz SF, Scheef $\mathrm{L}$, Boecker $\mathrm{H}$, et al. MRI follow-up after magnetic resonance-guided focused ultrasound for non-invasive thalamotomy: the neuroradiologist's perspective. Neuroradiology. 2020 May;62(9):1111-22.

67 Wintermark M, Druzgal J, Huss DS, Khaled MA, Monteith S, Raghavan P, et al. Imaging findings in MR imaging-guided focused ultrasound treatment for patients with essential tremor. AJNR Am J Neuroradiol. 2014 May;35(5):891-6.

68 Kinoshita M, McDannold N, Jolesz FA, Hynynen K. Noninvasive localized delivery of Herceptin to the mouse brain by MRIguided focused ultrasound-induced bloodbrain barrier disruption. Proc Natl Acad Sci U S A. 2006 Aug;103(31):11719-23.

69 McDannold N, Arvanitis CD, Vykhodtseva $\mathrm{N}$, Livingstone MS. Temporary disruption of the blood-brain barrier by use of ultrasound and microbubbles: safety and efficacy evaluation in rhesus macaques. Cancer Res. 2012 Jul;72(14):3652-63.
70 Stuckey SL, Goh TD, Heffernan T, Rowan D. Hyperintensity in the subarachnoid space on FLAIR MRI. AJR Am J Roentgenol. 2007 Oct;189(4):913-21.

71 Meng Y, Abrahao A, Heyn CC, Bethune AJ, Huang Y, Pople CB, et al. Glymphatics visualization after focused ultrasound-induced blood-brain barrier opening in humans. Ann Neurol. 2019 Dec;86(6):975-80.

72 Meng Y, MacIntosh BJ, Shirzadi Z, Kiss A, Bethune A, Heyn C, et al. Resting state functional connectivity changes after MR-guided focused ultrasound mediated bloodbrain barrier opening in patients with Alzheimer's disease. Neuroimage. 2019 Oct 15; 200:275-80.

73 Todd N, Zhang Y, Livingstone M, Borsook $\mathrm{D}, \mathrm{McD}$ annold $\mathrm{N}$. The neurovascular response is attenuated by focused ultrasoundmediated disruption of the blood-brain barrier. NeuroImage. 2019 Nov;201:116010.

74 Jung NY, Rachmilevitch I, Sibiger O, Amar T, Zadicario E, Chang JW. Factors related to successful energy transmission of focused ultrasound through a skull: a study in human cadavers and its comparison with clinical experiences. J Korean Neurosurg Soc. 2019 May;62(6):712-22.

75 GÂteau J, Marsac L, Pernot M, Aubry J-F, Tanter M, Fink M. Transcranial ultrasonic therapy based on time reversal of acoustically induced cavitation bubble signature. IEEE Trans Biomed Eng. 2010 Jan;57(1): 134-44.

76 O’Reilly MA, Hynynen K. A super-resolution ultrasound method for brain vascular mapping: super-resolution ultrasound method for brain vascular mapping. Med Phys. 2013 Oct;40(11):110701.

77 Davidson B, Hamani C, Rabin JS, Goubran $M$, Meng Y, Huang Y, et al. Magnetic resonance-guided focused ultrasound capsulotomy for refractory obsessive compulsive disorder and major depressive disorder: clinical and imaging results from two phase I trials. Mol Psychiatry. 2020 May;25(9): 1946-57.

78 Weidman EK, Kaplitt MG, Strybing K, Chazen JL. Repeat magnetic resonance imagingguided focused ultrasound thalamotomy for recurrent essential tremor: case report and review of MRI findings. J Neurosurg. 2020 Jan;132(1):211-6.

79 Shaw KD, Johnston AS, Rush-Evans S, Prather S, Maynard K. Nursing management of the patient undergoing focused ultrasound: a new treatment option for essential tremor. J Neurosci Nurs. 2017 Oct;49(5): 307-10.

80 Bjartmarz H, Rehncrona S. Comparison of accuracy and precision between framebased and frameless stereotactic navigation for deep brain stimulation electrode implantation. Stereotact Funct Neurosurg. 2007; 85(5):235-42.
Transcranial MR-Guided Focused Ultrasound
Stereotact Funct Neurosurg 2021;99:329-342 DOI: $10.1159 / 000512111$ 
81 Arvanitis CD, Livingstone MS, Vykhodtseva $\mathrm{N}$, McDannold N. Controlled ultrasoundinduced blood-brain barrier disruption using passive acoustic emissions monitoring. PLoS One. 2012;7(9):e45783.

82 Sun T, Samiotaki G, Wang S, Acosta C, Chen CC, Konofagou EE. Acoustic cavitationbased monitoring of the reversibility and permeability of ultrasound-induced bloodbrain barrier opening. Phys Med Biol. 2015 Dec;60(23):9079-94.

83 Cho EE, Drazic J, Ganguly M, Stefanovic B, Hynynen K. Two-photon fluorescence microscopy study of cerebrovascular dynamics in ultrasound-induced blood-brain barrier opening. J Cereb Blood Flow Metab. 2011 Sep;31(9):1852-62.
84 Nhan T, Burgess A, Cho EE, Stefanovic B, Lilge L, Hynynen K. Drug delivery to the brain by focused ultrasound induced bloodbrain barrier disruption: quantitative evaluation of enhanced permeability of cerebral vasculature using two-photon microscopy. J Control Release. 2013 Nov;172(1):274-80.

85 Park J, Zhang Y, Vykhodtseva N, Jolesz FA, McDannold NJ. The kinetics of blood brain barrier permeability and targeted doxorubicin delivery into brain induced by focused ultrasound. J Control Release. 2012 Aug; 162(1):134-42.
86 Yang FY, Ko CE, Huang SY, Chung IF, Chen GS. Pharmacokinetic changes induced by focused ultrasound in glioma-bearing rats as measured by dynamic contrast-enhanced MRI. PLoS One. 2014;9(3):e92910.

87 Arif WM, Elsinga PH, Gasca-Salas C, Versluis M, Martínez-Fernández R, Dierckx RAJO, et al. Focused ultrasound for opening blood-brain barrier and drug delivery monitored with positron emission tomography. J Control Release. 2020 Aug;324:303-16.

88 Focused Ultrasound Foundation. Brain mini workshop: treatment envelope expansion. Charlottesville, VA: The University of Virginia Darden School of Business; 2013. 